\title{
ON LOCAL MAXIMALITY FOR THE COEFFICIENT $a_{6}$
}

\author{
JAMES A. JENKINS and MITSURU OZAWA
}

Dedicated to Professor K. Noshiro on his 60th birthday

1. Recently a number of authors have studied the application of Grunsky's coefficient inequalities to the study of the Bieberbach conjecture for the class of normalized regular univalent functions $f(z)$ in the unit circle $|z|<1$

$$
f(z)=z+\sum_{n=2}^{\infty} a_{n} z^{n} .
$$

Charzynski and Schiffer [2] applied this result to give an elementary proof of the inequality $\left|a_{4}\right| \leqq 4$. One of the present authors [8] proved that if $a_{2}$ is real non-negative then $\Re a_{6} \leqq 6$. A natural first step in the study of the inequality for a coefficient is to prove local maximality for $a_{2}$ near to 2 . Bombieri [1] announced that he had proved

$$
\Re a_{6} \leqq 6-A\left(2-\Re a_{2}\right)
$$

for $A>0, \Re a_{2}$ sufficiently near to 2 . As yet to our knowledge no complete account of his result has appeared. One of the present authors has shown [7] that in many cases the Area Principle is more effective than Grunsky's method. In the present instance the Area Principle takes the form of an inequality due to Golusin [4]. In this paper we use this inequality to prove the local maximality of $\Re a_{6}$ at the Koebe function. Our theorem implies the result of Bombieri.

During the preparation of this work there appeared a paper by Garabedian, Ross and Schiffer [3] which asserts the local maximality of $\Re a_{2 n}, n=2,3, \ldots \ldots$ at the Koebe function. Further consideration is required to determine its status. In any case it does not appear to include Bombieri's result.

\section{Golusin's inequality and Grunsky's inequality.}

Let $f(z)$ be a normalized regular function univalent in the unit disc $|z|<1$, whose expansion around $z=0$ is

Received May 20, 1966.

The first author was supported in part by the National Science Foundation and the second author was supported by an N.S.F. Foreign Scientist Fellowship during 1965-66. 


$$
z+\sum_{\nu=2}^{\infty} a_{\nu} z^{\nu}
$$

Let $G_{\mu}(w)$ be the $\mu^{t h}$ Faber polynomial which is defined by

$$
\begin{gathered}
g_{\mu}(z)=G_{\mu}(g(z))=z^{\mu}+\sum_{\nu=1}^{\infty} \frac{b_{\mu \nu}}{z^{\nu}}, \\
g(z)=f\left(1 / z^{2}\right)^{-1 / 2} .
\end{gathered}
$$

Then it is known that $\nu b_{\mu \nu}=\mu b_{\nu \mu}$. Let

$$
Q_{m}(g(z))=\sum_{\mu=1}^{m} x_{\mu} g_{\mu}(z)
$$

then Golusin's inequality has the form

$$
\sum_{\nu=1}^{\infty} \nu\left|\sum_{\mu=1}^{m} x_{\mu} b_{\mu \nu}\right|^{2} \leqq \sum_{\nu=1}^{m} \nu\left|x_{\nu}\right|^{2},
$$

and Grunsky's inequality has the form

$$
\left|\sum_{\mu, \nu=1}^{m} \nu b_{\mu \nu} x_{\mu} x_{\nu}\right| \leqq \sum_{\nu=1}^{m} \nu\left|x_{\nu}\right|^{2}
$$

One of the authors [7] pointed out that Grunsky's inequality is a direct consequence of Golusin's.

By a simple calculation we have

$$
\begin{aligned}
& b_{11}=-\frac{1}{2} a_{2}, b_{13}=-\frac{1}{2}\left(a_{3}-\frac{3}{4} a_{2}^{2}\right), b_{15}=-\frac{1}{2}\left(a_{4}-\frac{3}{2} a_{2} a_{3}+\frac{5}{8} a_{2}^{3}\right), \\
& b_{17}=-\frac{1}{2}\left(a_{5}-\frac{3}{2} a_{2} a_{4}-\frac{3}{4} a_{3}^{2}+\frac{15}{8} a_{3} a_{2}^{2}-\frac{35}{64} a_{2}^{4}\right), \\
& b_{22}=-a_{3}+a_{2}^{2}, b_{24}=-a_{4}+2 a_{2} a_{3}-a_{2}^{3}, \\
& b_{44}=-2 a_{5}+4 a_{2} a_{4}-8 a_{2}^{2} a_{3}+3 a_{3}^{2}+3 a_{2}^{4}, \\
& b_{31}=-\frac{3}{2}\left(a_{3}-\frac{3}{4} a_{2}^{2}\right)=3 b_{13}, b_{33}=-\frac{3}{2}\left(a_{4}-2 a_{2} a_{3}+\frac{13}{12} a_{2}^{3}\right), \\
& b_{35}=-\frac{3}{2}\left(a_{5}-2 a_{2} a_{4}-\frac{5}{4} a_{3}^{2}+\frac{29}{8} a_{3} a_{2}^{2}-\frac{85}{64} a_{2}^{4}\right)=\frac{3}{5} b_{53}, \\
& b_{51}=5 b_{15}, b_{55}=-\frac{5}{2}\left(a_{6}-2 a_{2} a_{5}-3 a_{3} a_{4}+4 a_{2}^{2} a_{4}+\frac{21}{4} a_{2} a_{3}^{2}\right. \\
& \left.\quad-\frac{59}{8} a_{3} a_{2}^{3}+\frac{689}{320} a_{2}^{5}\right) .
\end{aligned}
$$


From now on we shall use the following notations:

$$
\begin{aligned}
2-x+i x^{\prime} & =a_{2}, \\
y+i y^{\prime} & =a_{3}-\frac{3}{4} a_{2}^{2}, \\
\eta+i \eta^{\prime} & =a_{4}-\frac{3}{2} a_{2} a_{3}+\frac{5}{8} a_{2}^{3}, \\
\xi+i \xi^{\prime} & =a_{5}-\frac{3}{2} a_{2} a_{4}-\frac{3}{4} a_{3}^{2}+\frac{15}{8} a_{3} a_{2}^{2}-\frac{35}{64} a_{2}^{4} .
\end{aligned}
$$

\section{Lemmas.}

Lemma 1. $7\left(\xi^{2}+\xi^{\prime 2}\right)+5\left(\eta^{2}+\eta^{\prime 2}\right)+3\left(y^{2}+y^{\prime 2}\right) \leqq 4 x-x^{2}-x^{\prime 2}$.

Proof. This is a simple consequence of the area theorem for $f\left(1 / z^{2}\right)^{-1 / 2}$.

$$
\text { Lemma 2. } \quad y \leqq 3 x-\frac{15}{4} x^{2}+\frac{10}{3} x^{3}-\frac{1}{4} x^{\prime 2} \text {. }
$$

Proof. One of the authors [6] proved the following result:

$$
\Re\left\{e^{-2 i \emptyset}\left(a_{3}-\frac{3}{4} a_{2}^{2}\right)\right\} \leqq 1+\frac{3}{8} \tau^{2}-\frac{\tau^{2}}{4} \log \frac{\tau}{4}+\frac{1}{4} \Re\left\{e^{-2 i \emptyset} a_{2}^{2}\right\}+\tau \Re\left\{e^{-i \Phi} a_{2}\right\}
$$

holds for every real $\Phi$ and for every real $\tau$ satisfying $0 \leqq \tau \leqq 4$.

Putting $\Phi=\pi$ and $\tau=4 e^{-s}$, we have

$$
y \leqq 2-8 e^{-s}+6 e^{-2 s}+4 s e^{-2 s}-x+\frac{x^{2}}{4}+4 x e^{-s}-\frac{1}{4} x^{\prime 2} .
$$

By a similar discussion in [8] we have the desired result.

Lemma 3.

$$
-x+\frac{x^{2}}{4}-x^{\prime 2} \leqq y .
$$

Proof. It is well-known that

$$
\Re\left(a_{2}^{2}-a_{3}\right) \leqq 1
$$

This implies the desired result.

LeMmA 4. $\quad \eta \leqq \frac{5}{4} x-\frac{3}{4} x^{2}+\frac{7}{48} x^{3}-\frac{1}{2} x^{\prime} y^{\prime}+\frac{x^{\prime 2}}{2}-\frac{x x^{\prime 2}}{4}$.

Proof. In (2) we select $m=3, x_{1}=\beta, x_{2}=0, x_{3}=1 / 3$. Then

$$
\left|a_{4}-2 a_{2} a_{3}+-13 a_{12}^{3}+2 \beta\left(a_{3}-\frac{3}{4} a_{2}^{2}\right)+\beta^{2} a_{2}\right| \leqq \frac{2}{3}+2|\beta|^{2} .
$$


Put $\beta=(2-x) / 4$ and take the real part. Then we have

$$
\begin{aligned}
\eta+\frac{1}{2} x^{\prime} y^{\prime}+\frac{1}{12} & \left.\left((2-x)^{3}-3(2-x) x^{\prime 2}\right)\right)+\frac{1}{16}(2-x)^{3} \\
& \leqq \frac{2}{3}+\frac{1}{8}(2-x)^{2}
\end{aligned}
$$

which is nothing but the desired result.

LeMmA 5. $-\eta \leqq \frac{1}{2}(2-x) y+2 x-\frac{3}{2} x^{2}+\frac{1}{3} x^{3}+\frac{1}{4}(2-x) x^{\prime 2}+\frac{1}{2} x^{\prime} y^{\prime}$.

Proof. One of the authors [6] proved the following fact:

$$
\Re\left\{e^{-3 i \emptyset}\left(-a_{4}+2 a_{2} a_{3}-a_{2}^{3}\right)\right\} \leqq \frac{2}{3}+2 \sigma^{2}+\frac{2}{3} \sigma^{3}+2 \sigma \Re\left\{e^{-2 i \emptyset}\left(a_{2}^{2}-a_{3}\right)\right\}
$$

for every real $\Phi$ and for every real $\sigma$ satisfying $-1 \leqq \sigma \leqq 1 / 3$.

Put $\Phi=0$ and $\sigma=-1+x / 2$. Then we have the desired result.

LEMma 6.

$$
\begin{aligned}
& (2-x) \eta-x^{\prime} \eta^{\prime}+\frac{3}{2}\left(y^{2}-y^{\prime 2}\right)-\frac{1}{2}\left((2-x)^{2}-x^{\prime 2}\right) y+(2-x) x^{\prime} y^{\prime} \\
& -2 x+\frac{3}{2} x^{2}-\frac{1}{2} x^{3}+\frac{1}{16} x^{4}-\frac{3}{8}(2+x)^{2} x^{\prime 2}+\frac{1}{16} x^{\prime 4} \leqq 2 \xi .
\end{aligned}
$$

Proof. By Grunsky's inequality we have

$$
\left|-2 a_{5}+4 a_{2} a_{4}-8 a_{2}^{2} a_{3}+3 a_{3}^{2}+3 a_{2}^{4}\right| \leqq 1 .
$$

This turns out to the desired inequality taking the real part.

Lemma 7. $\xi \leqq \frac{1}{2}(2-x) \eta-1 x^{\prime} \eta^{\prime}+\frac{1}{4}\left(y^{2}-y^{\prime 2}\right)+\frac{1}{2}+3 e^{-4 x}$

$$
\begin{aligned}
& +4 x e^{-4 x}+4 e^{-2 x}\left(y-\frac{1}{4}(2-x)^{2}+\frac{1}{4} x^{\prime 2}\right) \\
& +\frac{1}{2}\left(-y+\frac{1}{4}(2-x)^{2}-\frac{1}{4} x^{\prime 2}\right)^{2}-1\left(-y^{\prime}+\frac{1}{2}(2-x) x^{\prime}\right)^{2}
\end{aligned}
$$

for $0 \leqq x \leqq 2$.

Proof. One of the authors [6] proved the following result:

$$
\begin{aligned}
& -\Re\left\{e^{-4 i \Psi}\left(-a_{5}+2 a_{2} a_{4}+a_{3}^{2}-3 a_{2}^{2} a_{3}+a_{2}^{4}\right)\right\} \\
& \leqq-\frac{1}{2}+\frac{3}{16} \sigma^{4}-\frac{1}{8} \sigma^{4} \log \frac{\sigma^{2}}{4}+\frac{1}{2} \Re\left\{e^{-4 i \Psi}\left(a_{2}^{4}-a_{3}\right)^{2}\right\}-\sigma^{2} \Re\left\{e^{-2 i \Psi}\left(a_{2}^{2}-a_{3}\right)\right\}
\end{aligned}
$$

for $\Psi$ real and $0 \leqq \sigma \leqq 2$. Put $\Psi=0$ and $\sigma=2 e^{-x}, 0 \leqq x \leqq 2$. 
Then a simple calculation leads to the desired result.

It should be remarked that for $x \rightarrow 0$

$$
y=O(x), \quad \eta=O(x), \xi=O(x),
$$

and

$$
x^{\prime}=O\left(x^{1 / 2}\right), \quad y^{\prime}=O\left(x^{1 / 2}\right), \quad \eta^{\prime}=O\left(x^{1 / 2}\right), \quad \xi^{\prime}=O\left(x^{1 / 2}\right) .
$$

So far as local maximality is concerned we can consider only terms of order $O(x)$. Hence we shall omit terms of higher order in the sequel.

4. By the Golusin inequality we have

$$
\begin{gathered}
5\left|x_{5} b_{55}+x_{3} b_{35}+x_{1} b_{15}\right|^{2}+3\left|x_{5} b_{53}+x_{3} b_{33}+x_{1} b_{13}\right|^{2}+\left|x_{5} b_{51}+x_{3} b_{31}+x_{1} b_{11}\right|^{2} \\
\\
\leqq\left|x_{1}\right|^{2}+3\left|x_{3}\right|^{2}+5\left|x_{5}\right|^{2} .
\end{gathered}
$$

Put $x_{5}=1, x_{3}=5 \beta / 6, x_{1}=5 \delta$. Then we have

$$
\begin{array}{ll} 
& \mid a_{6}-2 a_{2} a_{5}-3 a_{3} a_{4}+4 a_{2}^{2} a_{4}+\frac{21}{4} a_{2} a_{3}^{2}-\frac{59}{8} a_{3} a_{2}^{3}+\frac{689}{320} a_{2}^{5} \\
& +\frac{1}{2}\left(a_{5}-2 a_{2} a_{4}-\frac{5}{4} a_{3}^{2}+\frac{29}{8} a_{3} a_{2}^{2}-\frac{85}{64} a_{2}^{4}\right) \beta+\left.\left(a_{4}-\frac{3}{2} a_{2} a_{3}+\frac{5}{8} a_{2}^{3}\right) \delta\right|^{2} \\
(3) \quad+\frac{3}{5} \mid a_{5}-2 a_{2} a_{4}-\frac{5}{4} a_{3}^{2}+\frac{29}{8} a_{3} a_{2}^{2}-\frac{85}{64} a_{2}^{4}+\frac{1}{2}\left(a_{4}-2 a_{2} a_{3}+\frac{13}{12} a_{2}^{3}\right) \beta \\
+\left.\left(a_{3} \frac{3}{4} a_{2}^{2}\right) \delta\right|^{2}+\frac{1}{5}\left|a_{4}-\frac{3}{2} a_{2} a_{3}+\frac{5}{8} a_{2}^{3}+\frac{1}{2}\left(a_{3}-\frac{3}{4} a_{2}^{2}\right) \beta+a_{2} \delta\right|^{2} \\
\quad \leqq \frac{4}{25}+\frac{1}{15}|\beta|^{2}+\frac{4}{5}|\delta|^{2} .
\end{array}
$$

Put $x_{5}=0, x_{3}=2 / 3, x_{1}=2 \beta$. Then we have

$$
\begin{aligned}
& 5\left|a_{5}-2 a_{2} a_{4}-\frac{5}{4} a_{3}^{2}+\frac{29}{8} a_{3} a_{2}^{2}-\frac{85}{64} a_{2}^{4}+\left(a_{4}-\frac{3}{2} a_{2} a_{3}+\frac{5}{8} a_{2}^{3}\right) \beta\right|^{2} \\
& (4) \quad+3\left|a_{4}-2 a_{2} a_{3}+\frac{13}{12} a_{2}^{3}+\left(a_{3}-\frac{3}{4} a_{2}^{2}\right) \beta\right|^{2}+\left|a_{3}-\frac{3}{4} a_{2}^{2}+a_{2} \beta\right|^{2} \\
& \quad \leqq \frac{4}{3}+4|\beta|^{2}
\end{aligned}
$$

From (4) we have, omitting higher order terms,

$$
\begin{aligned}
\eta+y(2 \beta-1) & \leqq\left(1+\beta^{2}\right) x+\frac{1}{2} x^{\prime 2}-\frac{1}{2} x^{\prime} y^{\prime}-\frac{1}{4}\left(y^{\prime}+\beta x^{\prime}\right)^{2} \\
& -\frac{3}{4}\left(\eta^{\prime}+(\beta-1) y^{\prime}+x^{\prime}\right)^{2}-\frac{5}{4}\left(\xi^{\prime}+(\beta-1) \eta^{\prime}+y^{\prime}\right)^{2}
\end{aligned}
$$


with real $\beta$. Put $\beta=5 / 2$. Then

$$
\begin{aligned}
\eta+4 y \leqq \frac{29}{4} x+\frac{1}{2} x^{\prime 2} & -\frac{1}{2} x^{\prime} y^{\prime}-\frac{1}{4}\left(y^{\prime}+\frac{5}{2} x^{\prime}\right)^{2} \\
& -\frac{3}{4}\left(\eta^{\prime}+\frac{3}{2} y^{\prime}+x^{\prime}\right)^{2}-\frac{5}{4}\left(\xi^{\prime}+\frac{3}{2} \eta^{\prime}+y^{\prime}\right)^{2} .
\end{aligned}
$$

From (3) putting $\beta=4$ and $\delta=2.25$ and omitting higher order terms, we have

$$
\begin{aligned}
\Re a_{6} & \leqq 6+0.5 \eta+2 y-\left(10-2.25^{2}\right) x-12 x^{\prime 2}-14.5 x^{\prime} y^{\prime}-3.5 y^{\prime 2} \\
& -7 x^{\prime} \eta^{\prime}-3 y^{\prime} \eta^{\prime}-2 x^{\prime} \xi^{\prime} \\
& -3\left(\xi^{\prime}+\eta^{\prime}+1.25 y^{\prime}+2 x^{\prime}\right)^{2}-\frac{1}{4}\left(\eta^{\prime}+2 y^{\prime}+2.25 x^{\prime}\right)^{2} .
\end{aligned}
$$

By (5) we have

$$
\begin{aligned}
\Re a_{6} & \leqq \\
& -2 x^{\prime} \xi^{\prime}-\frac{3}{4}\left(\xi^{\prime}+\eta^{\prime}+1.25 y^{\prime}+2 x^{\prime}\right)^{2} \\
& -\frac{1}{4}\left(\eta^{\prime}+2 y^{\prime}+2.25 x^{\prime}\right)^{2}-\frac{1}{8}\left(y^{\prime}+\frac{5}{2} x^{\prime}\right)^{2}-\frac{3}{8}\left(\eta^{\prime}+\frac{3}{2} y^{\prime}+x^{\prime}\right)^{2} \\
& -\frac{5}{8}\left(\xi^{\prime}+\frac{3}{2}-\eta^{\prime}+y^{\prime}\right)^{2} .
\end{aligned}
$$

Since $x^{\prime 2}+3 y^{\prime 2}+5 \eta^{\prime 2}+7 \xi^{\prime 2} \leqq 4 x$ omitting higher order terms in Lemma 1 , we have

(7) $\Re a_{6} \leqq 6-F\left(x^{\prime}, y^{\prime}, \eta^{\prime}, \xi^{\prime}\right)$,

$$
\begin{aligned}
F & \left(x^{\prime}, y^{\prime}, \eta^{\prime}, \xi^{\prime}\right)=\left(11.75+\frac{1.3125}{4}\right) x^{\prime 2}+14.75 x^{\prime} y^{\prime}+\left(3.5+\frac{3 \times 1.3125}{4}\right) y^{\prime 2} \\
& +7 x^{\prime} \eta^{\prime}+3 y^{\prime} \eta^{\prime}+\frac{5 \times 1.3125}{4} \eta^{\prime 2}+2 x^{\prime} \xi^{\prime}+\frac{7}{4}-1.3125 \xi^{\prime 2} \\
& +\frac{3}{4}\left(\xi^{\prime}+\eta^{\prime}+1.25 y^{\prime}+2 x^{\prime}\right)^{2}+\frac{1}{4}\left(\eta^{\prime}+2 y^{\prime}+2.25 x^{\prime}\right)^{2}+\frac{1}{8}\left(y^{\prime}+\frac{5}{2} x^{\prime}\right)^{2} \\
& +\frac{3}{8}\left(\eta^{\prime}+\frac{3}{2} y^{\prime}+x^{\prime}\right)^{2}+\frac{5}{8}\left(\xi^{\prime}+\frac{3}{2} \eta^{\prime}+y^{\prime}\right)^{2} .
\end{aligned}
$$

Now we shall prove the positive definiteness of $F\left(x^{\prime}, y^{\prime}, \eta^{\prime}, \xi^{\prime}\right)$. Consider $64 F\left(x^{\prime}, y^{\prime}, \eta^{\prime}, \xi^{\prime}\right)$. This is equal to

$$
\begin{gathered}
1120 x^{\prime 2}+1440 x^{\prime} y^{\prime}+528 y^{\prime 2}+760 x^{\prime} \eta^{\prime}+568 y^{\prime} \eta^{\prime}+283 \eta^{2} \\
+320 x^{\prime} \xi^{\prime}+200 y^{\prime} \xi^{\prime}+216 \eta^{\prime} \xi^{\prime}+235 \xi^{\prime 2} .
\end{gathered}
$$

Consider the principal diagonal minor determinants 


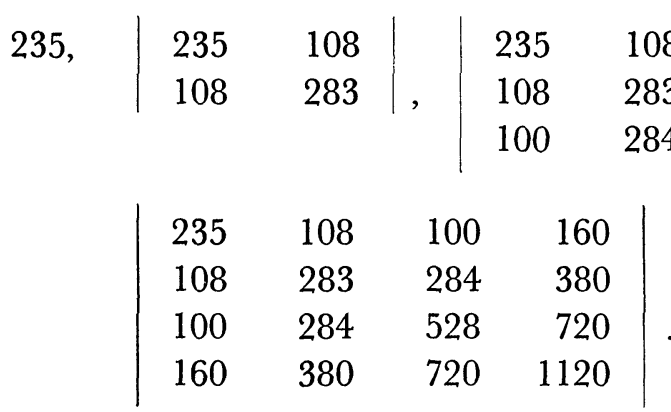

Then these are positive. Hence $64 F\left(x^{\prime}, y^{\prime}, \eta^{\prime}, \xi^{\prime}\right)$ is positive definite. By continuity we have

$$
\Re a_{6} \leqq 6-A x-Q\left(x^{\prime}, y^{\prime}, \eta^{\prime}, \xi^{\prime}\right)
$$

with a suitable positive $A$ and a suitable positive definite quadratic form $Q\left(x^{\prime}, y^{\prime}, \eta^{\prime}, \xi^{\prime}\right)$. This implies the following theorem.

THEOREM. Let $f(z)$ be a normalized regular function univalent in $|z|<1$, whose local expansion is

$$
z+\sum_{\nu=2}^{\infty} a_{\nu} z^{\nu}
$$

Then

$$
\Re a_{6} \leqq 6-A x, A>0
$$

holds for $0 \leqq x<\varepsilon$. If $\Re a_{6}=6$ in $0 \leqq x<\varepsilon$, then $f(z)$ reduces to the Koebe function

$$
\frac{z}{(1-z)^{2}}
$$

\section{REFERENCES}

[1] Bombieri, E., Sul problema di Bieberbach per le funzioni univalenti. Rend. Lincei 35 (1963), 469-471.

[2] Charzinski, Z. and M. Schiffer, A new proof of the Bieberbach conjecture for the fourth coefficient. Arch Rat. Mech. Anal. 5 (1960), 187-193.

[3] Garabedian, P.R., G.G. Ross and M. Schiffer, On the Bieberbach conjecture for even n. Journ. Math. Mech. 14 (1965), 975-989.

[4] Golusin, G.M., On p-valent functions. Mat. Sbornik (N.S.) 8 (1940), 277-284.

[5] Grunsky, H., Koeffizientenbedingungen für schlicht abbildende Funktionen. Math. Zeits. 45 (1939), 29-61.

[6] Jenkins, J.A., On certain coefficients of univalent functions. Analytic functions. Princeton Univ. Press (1960), 159-194.

[7] Some area theorems and a special coefficient theorem. Illinois Journ. Math. 8 (1964), 80-99. 
[8] Ozawa, M., On the sixth coefficient of univalent function. Kōdai Math. Sem. Rep. 17 (1965), 1-9.

Washington University, St. Louis and Tokyo Institute of Technology, Tokyo. 